OPEN ACCESS

Edited by:

Cheng Zhong,

Tianjin University, China

Reviewed by:

Yunhua Xu,

Tianjin University, China

Fang Fang,

Fudan University, China

${ }^{*}$ Correspondence: Rengapillai Subadevi susimsk@yahoo.co.in

Fu-Ming Wang

mccabe@mail.ntust.edu.tw

Marimuthu Sivakumar

susiva73@yahoo.co.in

Specialty section:

This article was submitted to

Energy Materials,

a section of the journal

Frontiers in Materials

Received: 24 February 2020

Accepted: 08 April 2020

Published: 29 May 2020

Citation:

Kalaiselvi C, Subadevi R, Wang F-M and Sivakumar M (2020) Sepiolite

Enfolded Sulfur/ZnO Binary

Composite Cathode Material for Li-S

Battery. Front. Mater. 7:109.

doi: 10.3389/fmats.2020.00109

\section{Sepiolite Enfolded Sulfur/ZnO Binary Composite Cathode Material for Li-S Battery}

\author{
Chelladurai Kalaiselvi ${ }^{1}$, Rengapillai Subadevi ${ }^{1 *}$, Fu-Ming Wang ${ }^{2 *}$ and \\ Marimuthu Sivakumar ${ }^{1 *}$
}

${ }^{1}$ Energy Materials Lab, Department of Physics, Science Block, Alagappa University, Karaikudi, India, ${ }^{2}$ Graduate Institute of Applied Science and Technology, National Taiwan University of Science and Technology, Taipei, Taiwan

Lithium-Sulfur (Li-S) batteries have become an incredible challenge for the approaching age of high-vitality stockpiling gadgets. Nevertheless, one of the main specialized issues emerging in Li-S batteries is the polysulfide disintegration among electrodes and striking capacity limit. Here, sieved sepiolite (200 mesh sieve) has been favored as an added substance material to counter the above difficulties in the sulfur battery. The zinc oxide nanoparticles were prepared by co-precipitation method and a Sulfur/post-treated sieved Sepiolite/Zinc Oxide (S/PTSp/ZnO) nanocomposite was prepared by warmth treatment. The prepared composite was described by XRD, FTIR, RAMAN, SEM with EDAX, TEM, XPS, TG, and Electrochemical analysis. The S/PTSp/ZnO composite cathode exhibited an initial discharge capacity of $1,391 \mathrm{mAng}^{-1}$ and attained a stable capacity of 765 mAhg $^{-1}$ at 200 cycles. In conclusion, Li-S batteries can be relied upon to capably build up the utilization of sulfur and limit the disintegration of polysulfides into an electrolyte.

Keywords: sulfur, cathode, sepiolite, ZnO nanocomposite, Li-S battery, energy storage

\section{INTRODUCTION}

Sulfur offers one of the most noteworthy theoretical specific capacities $\left(1,675 \mathrm{mAhg}^{-1}\right)$ among all the metal oxides. The system could hypothetically deliver the energy density of 2,600 Wh $\mathrm{kg}^{-1}$ (Bruce et al., 2012). In addition, sulfur is an inexhaustible component, non-dangerous, and incredibly modest, which makes it appealing for substantial scale applications (Ji and Nazar, 2010). A realistic target of practical energy density is in the range of $400-600 \mathrm{Wh} \mathrm{kg}^{-1}$, which is higher when compared with the classical Li-ion batteries.

Li-S batteries are considered to be sensible for the generation of high-capacity energy storage devices.

However, there is still a long way to go and many challenges to overcome, since the system suffers from several unresolved drawbacks, which induce a large gap between reality and expectations. The major barriers to developing practical sulfur cathodes during the charge-discharge process include the highly insulating nature of sulfur, polysulfide dissolution, and volume expansion (Krishnaveni et al., 2018). The significant hindrances to the improvement of Li-S batteries are their low active material utilization rate, poor cycle life, and low coulombic efficiency (Evers and Nazar, 2013; Fu and Su, 2013). It is important to depict the method for how Li-S batteries work, as its mind-boggling working component is altogether different from the traditional Li-ion batteries. There are two basic strategies to conquer the above mentioned obstacles: (i) by coating the battery with metallic oxides and polymers; and (ii) by coating the battery with carbon materials, which could 
confine the sulfur or lithium polysulfides within the framework by using host materials (Radhika et al., 2018). A few methodologies have been connected to address the difficulties portrayed above, including the utilization of metal sulfides (Han et al., 2003), organic sulfides (Qiu et al., 2010; Wu et al., 2011), and metal oxides (Song et al., 2004; Zheng et al., 2006). Despite the fact that these techniques are helpful in improving the electrochemical performances, the security issues and sulfur as a functioning material can be utilized for electrode preparation. A conductive added substance is constantly added to the electrode, so as to give an electronic permeation to the entire electrode. Here post-treated sieved sepiolite acts as an additive material and it is well-matched for the establishment of a cathode material for Li-S batteries.

Sepiolite is a fibrous hydrated magnesium silicate clay mineral, having the elemental formula $\mathrm{Mg}_{8} \mathrm{Si}_{12} \mathrm{O}_{30}(\mathrm{OH})_{4}\left(\mathrm{H}_{2} \mathrm{O}\right)_{4} \cdot 8 \mathrm{H}_{2} \mathrm{O}$. Like other silicate minerals, it contains a hysterically twodimensional tetrahedral sheet but has no lasting octahedral sheets (Alvarez et al., 1984). In this manner, sepiolite has nano-sized channels containing water (Bokobza et al., 2004; Burzo and Wijn, 2009). The fibrous naturally visible high specific surface region, magnificent adsorption properties, rich micro- porosity, and low cost are additionally outstanding properties of the sepiolite material (Can et al., 2010; Fu et al., 2015; Zhang et al., 2015). Based on the above highlights, Sepiolite has been given significantly more focus in the world and many researchers are looking to use clay minerals in electrode material research.

$\mathrm{ZnO}$ nanopowders have been synthesized using various methods, such as Moezzi et al. (2012) the soft chemical method (Vayssieres, 2003), the sol-gel process (Zhang et al., 2003), the vapor phase growth (Sun et al., 2004), the co-precipitation method, and the vapor liquid-solid process (Gao and Wang, 2004), and so forth. Here, zinc oxide is a non-poisonous, ntype II-VI semiconductor material having a wurtzite structure with a direct and wide band gap of $3.37 \mathrm{eV}$. Its properties include close UV emanation, optical straightforwardness, great electrical conductivity, and piezoelectricity (Ozgur et al., 2005; Coleman and Jagadish, 2006). The improved electrochemical performance was observed due to the good electrode/electrolyte contact ensured by the large surface area of nanoscale particles (Muruganantham et al., 2016). Sulfur acts as an insulator; for that reason the additive material, such as post-treated sieved sepiolite, and metal oxide $\mathrm{ZnO}$ nanoparticles are used to improve the conductivity of the material in the Li-S batteries.

\section{MATERIALS AND METHODS}

Sepiolite powders (Sp) were bought from a mineral processing factory in Sigma Aldrich, India. Sodium Hydroxide $(\mathrm{NaOH})$, Sulfur (S), Zinc oxide ( $\mathrm{ZnO})$, Zinc Nitrate Hexahydrate [ $\mathrm{Zn}$ $\left.\left(\mathrm{NO}_{3}\right)_{2} \cdot 6 \mathrm{H}_{2} \mathrm{O}\right]$, and Hydrochloric acid $(\mathrm{HCl})$, were purchased from Axiom Chemicals Private Limited (India). All chemicals of analytical grade were used without further purification.

\section{Synthesis of $\mathrm{ZnO}$ Nanoparticles}

Zinc oxide nanoparticles were synthesized by a co-precipitation method using zinc nitrate and sodium hydroxide as a precursor.

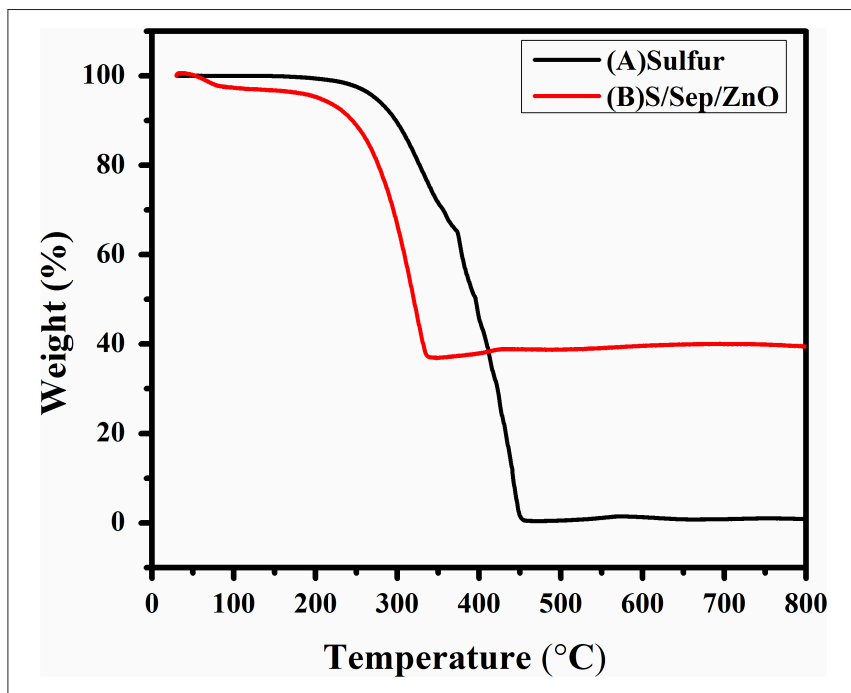

FIGURE 1 | TGA curves of (A) pure sulfur (B) S/Sp/ZnO.

In this experimental work, the $0.4 \mathrm{M}$ aqueous solution of zinc nitrate $\left[\mathrm{Zn}\left(\mathrm{NO}_{3}\right)_{2} \cdot 6 \mathrm{H}_{2} \mathrm{O}\right]$ was kept under constant stirring using a magnetic stirrer to completely dissolve the zinc nitrate for $1 \mathrm{~h}$ and $0.8 \mathrm{M}$ of $\mathrm{NaOH}$ aqueous solution was added drop by drop slowly under high-speed constant stirring. The reaction was allowed to proceed for $2 \mathrm{~h}$ after completion. The beaker was sealed after $4 \mathrm{~h}$. After the completion of the reaction, the solution was allowed to settle overnight and further, the supernatant solution was separated carefully. The remaining solution was centrifuged for $10 \mathrm{~min}$, and the precipitate was removed. Thus, precipitates of $\mathrm{ZnO}$ nanoparticles were cleaned with de-ionized water three times and methanol was used to remove byproducts and then dried at about $80^{\circ} \mathrm{C}$. Finally, dried $\mathrm{Zn}(\mathrm{OH})_{2}$ was completely converted into $\mathrm{ZnO}$ nanoparticles (Maensiri et al., 2006).

\section{Sepiolite Acid Treatment}

Acid treatment was carried out using sieved sepiolite powder by adding 8 mole ratio of Hydrochloric acid and de-ionized water in a beaker without distressing at the surrounding temperature for $24 \mathrm{~h}$. The clay minerals were filtered and washed with de-ionized water numerous times, and then dried at $40^{\circ} \mathrm{C}$ in a vacuum oven at $10^{-3}$ Torr for $12 \mathrm{~h}$.

\section{Thermal Activation Method}

The post-treated sieved sepiolite powder is further proceeded with thermal activation method. In this process, eight mole ratios of post-treated sepiolite and $\mathrm{ZnO}$ nanoparticles were ball milled for $1 \mathrm{~h}$ at normal speed, and then the sample was manually mixed with sublimed sulfur (7:2:1) through fine grinding for $1 \mathrm{~h}$ and heated at $155^{\circ} \mathrm{C}$ for $20 \mathrm{~h}$ in a muffle furnace. The collected sample was milled and dried to attain the final product.

\section{Characterization}

The X-ray diffraction patterns and functional groups of calcined powder samples were inspected using X-ray Diffraction 


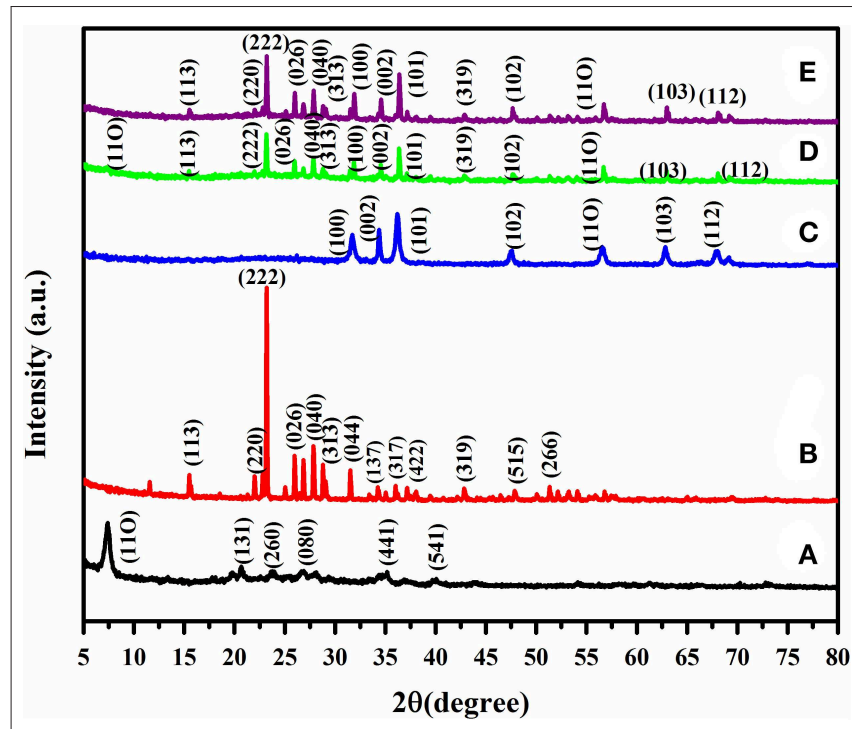

FIGURE 2 | XRD pattern of (A) SSp (seived sepiolite), (B) PS (sublimed sulfur), (C) ZnO (Zinc oxide nanoparticles), (D) S/SvSp/ZnO (sulfur/seived sepiolite/Zinc oxide), and (E) S/PTSp/ZnO (sulfur/post-treated seived sepiolite/Zinc oxide).

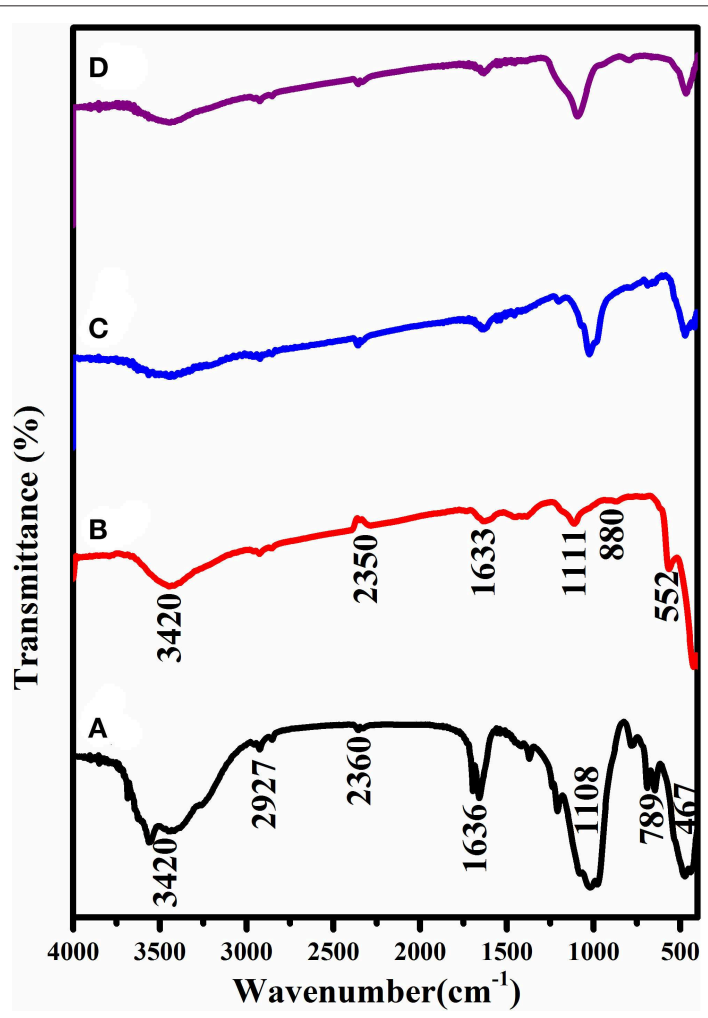

FIGURE 3 | FTIR spectra of (A) SSp, (B) ZnO, (C) S/SvSp/ZnO, and (D) S/PTSp/ZnO.

(PAN analytical XPERT-PRO with $\mathrm{Cu}-\mathrm{K} \alpha$ radiation) and FTIR spectrometer recording IR spectra in the scope of 4,000$400 \mathrm{~cm}^{-1}$ (Thermo Nicolet 380 Instrument Cooperation

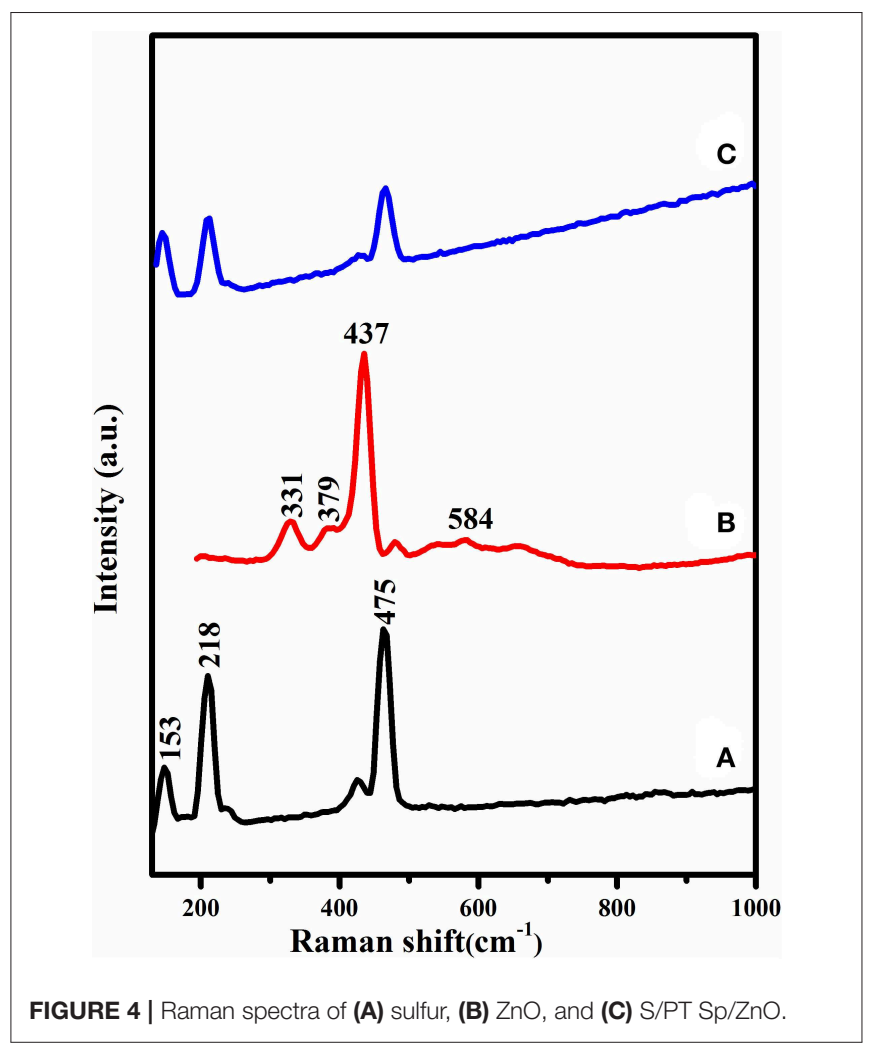

and $\mathrm{KBr}$ pellets). The morphology of the powders was reviewed by checking electron magnifying instrument (SEM FEG Quanta 250) and transmission electron magnifying lens (TEM) with vitality dispersive X-ray spectroscopy (EDS). The Thermogravimetric examination (TGA) estimations for the examples were done with a PerkinElmer Pyris Diamond TG/DTA warm analyzer in a nitrogen atmosphere with a warming rate of $10^{\circ} \mathrm{C} \mathrm{min}^{-1}$.

\section{Electrochemical Performance}

The positive electrodes comprised of $70 \mathrm{wt} \%$ of sulfur/sepiolite/ZnO composite, $20 \mathrm{wt} \%$ of acetylene black, and $10 \mathrm{wt} \%$ of polyvinylidene fluoride binders were homogeneously blended in an N-methyl-2-pyrrolidone (NMP) dissolvable and constantly ground for $3 \mathrm{~h}$. The slurry was consistently coated on an aluminum foil, and after that dried at $60^{\circ} \mathrm{C}$ for $12 \mathrm{~h}$. The CR2032 type coin cells were assembled in an argon-filled glove box, while lithium metal was utilized as a counter electrode and polypropylene layer was used as the separator in the Li-S battery. The electrolyte was prepared from 1,3-dioxolane, TEG (Tetra ethylene glycol), and DMG (dimethyl ether glycol). Electrochemical charge-release and cyclic performances were estimated at room temperature using a BTS-5V3A battery analyzer (Neware, Shenzhen, China), with the potential range from 3.0 to $1.0 \mathrm{~V}$ (vs. Li/Lis). Cyclic voltammetry (CV) and electrochemical impedance spectroscopy (EIS) were tested using a CHI660D electrochemical workstation (Chenhua, Shanghai, 

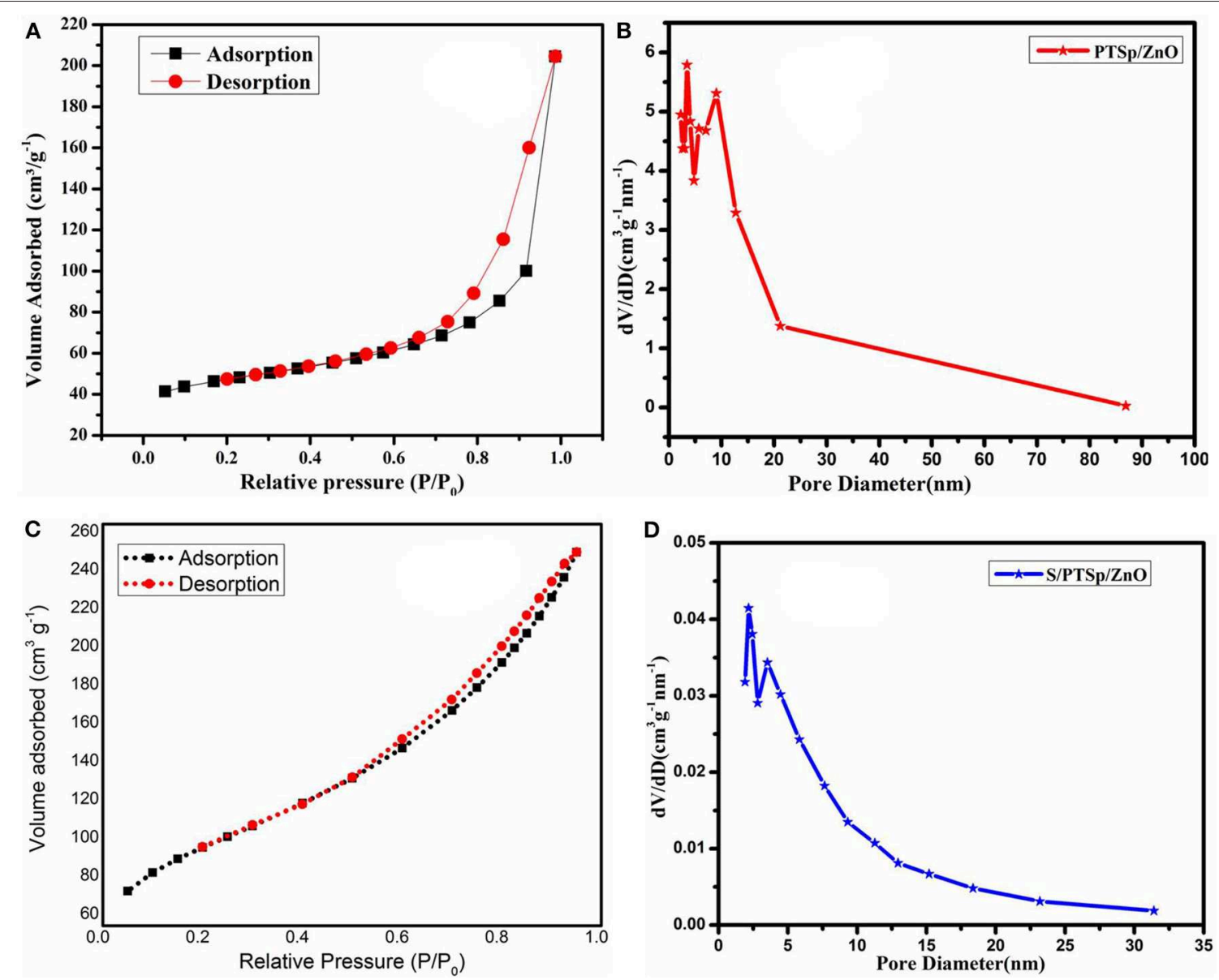

FIGURE 5 | Nitrogen adsorption-desorption isotherms at $77 \mathrm{~K}$ of (A,C) PT Sp/ZnO and S/PT Sp/ZnO, (B,D) BJH pore size distribution of PT Sp/ZnO and S/PT $\mathrm{Sp} / \mathrm{ZnO}$.

China) over a frequency range of $1 \mathrm{MHz}$ to $0.1 \mathrm{~Hz}$, with an opencircuit AC voltage amplitude of $5 \mathrm{mV}$.

\section{RESULTS AND DISCUSSION}

The thermal stability of the Sulfur and as-arranged $\mathrm{S} / \mathrm{Sp} / \mathrm{ZnO}$ composites have been investigated by Thermo gravimetric analysis (TG) under $\mathrm{N}_{2}$ atmosphere, as shown in Figure 1. At the point when the temperature increased, the mass of sulfur diminished to zero when it reached up to $315^{\circ} \mathrm{C}$, and this is attributed to the complete melting of sulfur. Both TGA curves of sulfur and $\mathrm{S} / \mathrm{Sp} / \mathrm{ZnO}$ composite demonstrates the weight reduction stages from around 200 to $315^{\circ} \mathrm{C}$, mirroring the evaporation of sulfur. The subsequent weight reduction of the composite is just about $65 \%$, because of the vanishing of sulfur which is consistent with the added amount of proportions ( $\mathrm{Li}$ et al., 2013; Yang et al., 2015).

The crystal structure of the as prepared sample was inspected by X-Ray Diffraction analysis and has been illustrated in Figure 2. The XRD pattern expressly shows that the as prepared zinc oxide has a leading peak at $2 \theta=31.64,34.36,36.22,47.55$, $56.54,62.79,66.2^{\circ}$ correlated to the Miller indices (100), (002), (101), (102), (110), (103), (112), respectively. This pattern is well in accordance with the hexagonal wurtzite period of $\mathrm{ZnO}$ (JCPDS Card No. 36-1451) through the sieved sepiolite reflection peak which appeared at $1.17 \mathrm{~nm}$, relating to the (110) peak that was observed. Due to thermal treatment, the melting sulfur has very low viscosity and could easily disperse into the porous substrates. After the sulfur was integrated into the porous spaces, the sulfur was well-wrapped up into the composite material, which suggests that no sulfur envelops the outer surface of the composite (Rajkumar et al., 2018). The well-resolved sulfur peaks were scattered in the covering materials (Zegeye et al., 2016) and composites showed peaks of sulfur with decreased peak intensity when contrasted with the bare sulfur (JCPDS-Card No. 77-0145), demonstrating that sulfur is in the mesoporous sepiolite material.

The functional groups of the FTIR spectra of S/PTSp/ZnO are as shown in Figure 3. There are several characteristic absorption peaks presented in the FT-IR spectra analysis. The bands at $1,108 \mathrm{~cm}^{-1}$ are allocated to $\mathrm{Si}-\mathrm{O}-\mathrm{Si}$ (silanol group) in-plane vibration. In this way, these peaks could be utilized to confirm the sepiolite clay mineral. The peak at $467 \mathrm{~cm}^{-1}$ is the characteristic 


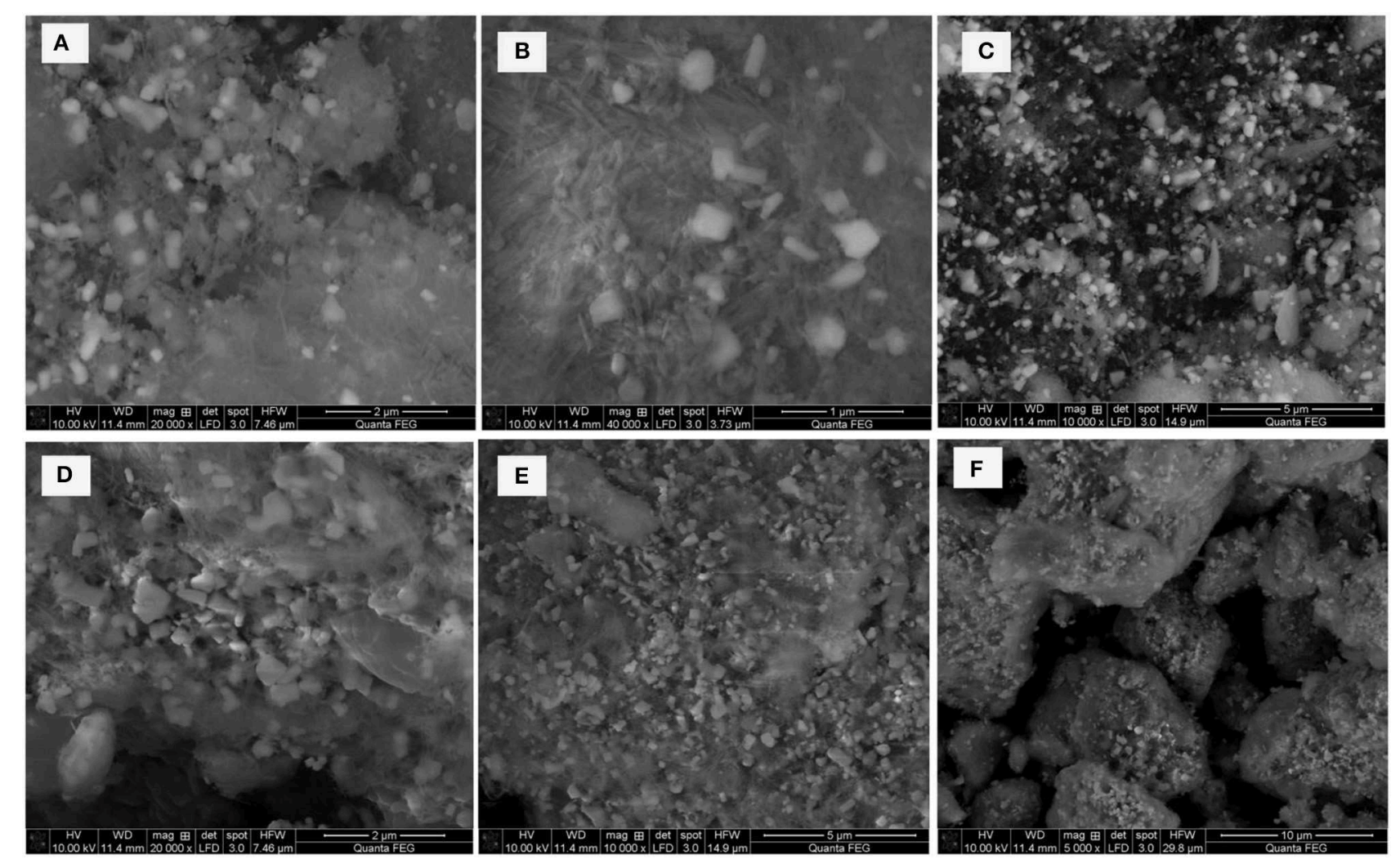

FIGURE 6 | SEM images of (A,B,C) PTSp/ZnO, (D,E,F) S/PTSp/ZnO.
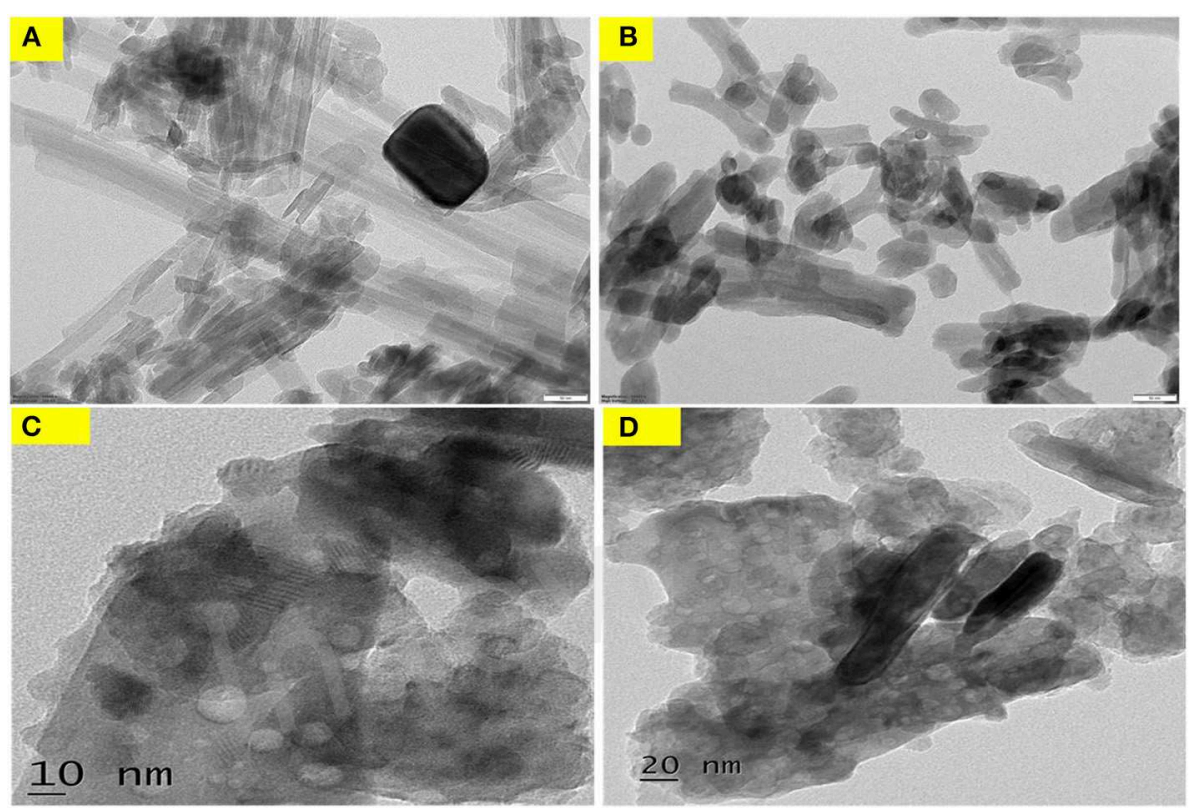

FIGURE 7 | TEM images of (A,B) PTSp/ZnO, (C,D) S/PTSp/ZnO.

absorption peaks of the wurtzite period of $\mathrm{Zn}$-O. These results verify the event of $\mathrm{Zn}-\mathrm{O}$ groups. $3,420 \mathrm{~cm}^{-1}$ can be credited to the $\mathrm{O}-\mathrm{H}$ stretching of the water. $2,927 \mathrm{~cm}^{-1}$ are allocated to the
$\mathrm{C}-\mathrm{H}$ asymmetric stretching vibration. $1,636 \mathrm{~cm}^{-1}$ are entrusted to the $\mathrm{C}=\mathrm{O}$ extending vibration. The $\mathrm{Si}-\mathrm{O}$ and $\mathrm{O}-\mathrm{H}$ groups can be clearly observed in the FTIR of S/PTSp/ZnO. 

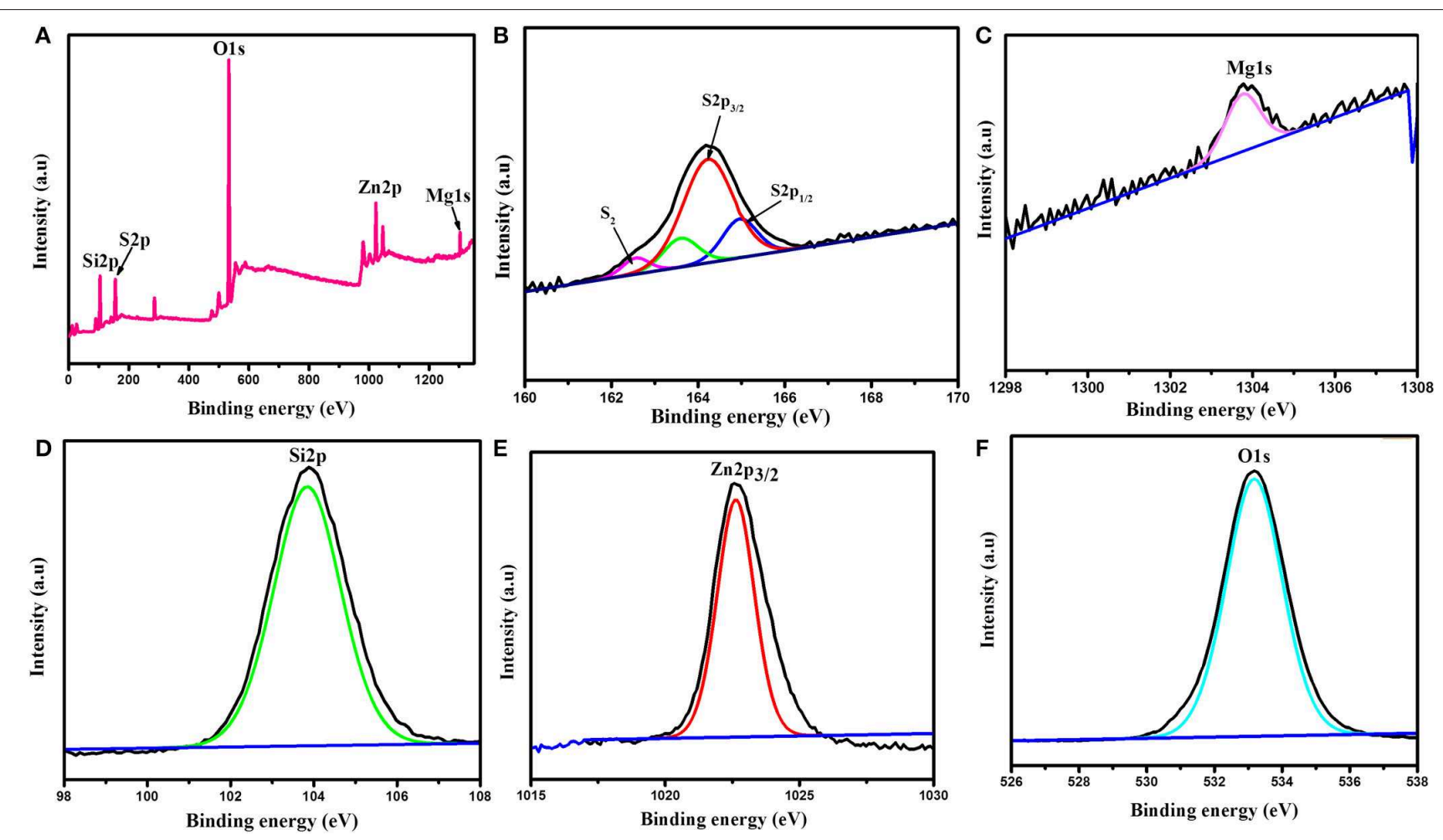

FIGURE 8 |XPS (A) Wide spectrum of S/PTSp/ZnO composite, Spectra of (B) S2p1/2 and S2P3/2, (C) Magnesium, (D) Silica, (E) Zn2p3/2, (F) Oxygen.

The Raman spectrums of sulfur, Zinc oxide, and S/PT $\mathrm{Sp} / \mathrm{ZnO}$ have appeared in Figure 4. The E2 mode situated at $437 \mathrm{~cm}^{-1}$ was observed for both compositions and showed the great crystallization nature of the wurtzite structure of $\mathrm{ZnO}$ nanoparticles. The band at $584 \mathrm{~cm}^{-1}$ is related to $\mathrm{E} 1$ mode. It is commonly acknowledged that E1 is identified with the development of imperfections in the nanoparticles. The pure sulfur displays a characteristic peak at underneath $500 \mathrm{~cm}^{-1}$, however here the peaks appeared nearly at 153,218, and 475 $\mathrm{cm}^{-1}$ which are started from the A1 symmetry mode of the S$\mathrm{S}$ bond (Ward, 1968). Raman spectra of S/PTSp/ZnO quickens some vibrational signals beneath $500 \mathrm{~cm}^{-1}$, showing that the sulfur is in a crystalline state. This outcome is in great agreement with the XRD results (Li et al., 2014).

Figure 5 displays the N2 adsorption-desorption isotherms plot which confirms the porous structure of the composite material. The hysteresis loop of the PTSp/ZnO and S/PTSp/ZnO composite indicates that the sample is mesoporous in nature. The shape of the isotherm is linked with type $1 \mathrm{~V}$ isotherms with $\mathrm{H} 3$ hysteresis of the IUPAC classification. Based on DFT calculations, the pore size distribution is mainly focused at about $2-5 \mathrm{~nm}$, which belongs to a mesoporous structure. The PTSp/ZnO sample exhibits a surface area of $320.5584 \mathrm{~m}^{2} \mathrm{~g}^{-1}$ and a pore volume of about $0.4 \mathrm{~cm}^{3} \mathrm{~g}^{-1}$, and also the S/PTSp/ZnO composite shows a specific surface of $172.603 \mathrm{~m}^{2} \mathrm{~g}^{-1}$ and a specific pore volume of $0.274 \mathrm{~cm}^{3} \mathrm{~g}^{-1}$. After sulfur impregnation, such a remarkable hysteresis loop was decreased and it was confirmed that the well distribution of sulfur particles had been embedded into the composite cathode material.

SEM analyses were carried out for all composite materials to investigate the structure and morphology on the surface of sample. Figures 6A-C demonstrates the different magnifications of the SEM picture of PT Sp/ZnO and Figures 6D-F shows the SEM images of S/PTSp/ZnO. From that picture, the different sizes of unruffled sporadic porous particles are exhibited in an as-prepared sample. The morphology of sepiolite powders is composed of small micro fibrous porous bundles and, furthermore, $\mathrm{ZnO}$ nano-composites are placed as consolidated on the sepiolite structure. After sulfur was infused, the particles uniformly dispersed and observable aggregations appeared owing to thermal treatment (Figure S1).

The TEM micrographs can be seen from Figures 7A-D. The TEM picture demonstrates that the nanocomposite of Zinc oxide comprises of little round particles and a dark pole-like shape that corresponds to that sieved sepiolite powder, and that both are consolidated on the surface of sublimed sulfur. $\mathrm{ZnO}$ nanoparticles alone exceedingly and consistently mono dispersed on the sepiolite. This uniform scattering is in accordance with the smaller pore estimate appropriation of $\mathrm{ZnO}-\mathrm{N}$ TEM images in figure. The fringes seen in Figure 7D explained the regular arrangement of atoms in the material.

XPS examinations have been employed from the survey of $0-$ $1,250 \mathrm{eV}$ to examine the S/PTSp/ZnO composite sample, which exposes the presence of $\mathrm{Mg}, \mathrm{Zn}, \mathrm{O}, \mathrm{C}, \mathrm{S}$, and $\mathrm{Si}$ elements. 


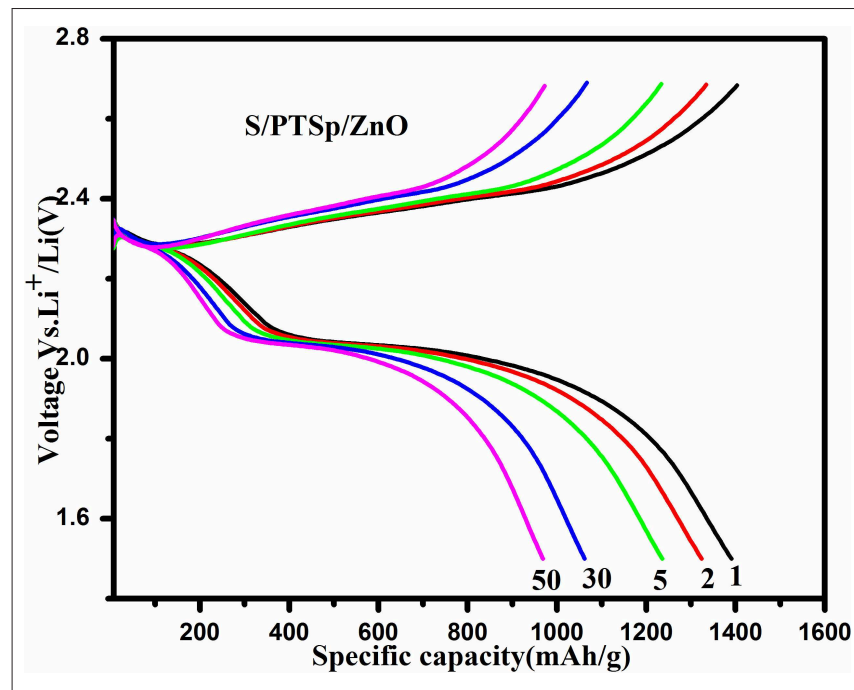

FIGURE 9 | Cyclic voltammetry curves of the S/PTSp/ZnO composite potential range from 1.5 to $3 \mathrm{~V}$ (verses $\mathrm{Li}^{+} / \mathrm{Li}$ ).

The peaks are situated at $1304.5,1022,532.6,285.4,164.1$, and $103.3 \mathrm{eV}$ corresponding to Mg1s, Zn2p, O1s, Cls, S2p, and Si2p, respectively. Figure $\mathbf{8}$ shows the wide spectrum of the as prepared sample which is attributed to of S2p1/2, S2p3/2, Mg1s, Si2p, $\mathrm{Zn} 2 \mathrm{p} 3 / 2$, and O1s. The peak appearing at 164.1 and $165.1 \mathrm{eV}$ correspond to S2p3/2 and S2p1/2a bridge sulfur of the polysulfide species, which is likely to be created by means of the oxidation of sulfur upon heating (Kumar et al., 2017). The short chain sulfur (S2) and sulfide (S22-) peaks are attributed to the S2p spectrum. The short chain sulfur (S2) and sulfide (S22-) peaks are attributed to the $S 2 \mathrm{p}$ spectrum in the range of 166 to $172 \mathrm{eV}$. The peak located at $167.2 \mathrm{eV}$ was ascribed to thiosulfate, which is formed due to polysulfide oxidation on the $\mathrm{ZnO}$ surface (Xu et al., 2015). The peak $1,022 \mathrm{eV}$ committed to zinc oxide nanocomposite particle.

\section{Electrochemical Properties}

The electrochemical mechanism of the as prepared composite lithium-sulfur battery was examined using cyclic voltammogram performances. Figure 9 illustrates the initial five cycles of the composite cathode material. The S/PTSp/ZnO composite reveals an anodic oxidation peak and two cathodic reduction peaks in the initial five cycles. These peaks are useful for a sulfurbased electrode. The two reduction peaks appearing at 2.29 and $2.0 \mathrm{~V}$, where the peak around $2.29 \mathrm{~V}$ is attributed to the pure sulfur, changed over into long-chain multi-lithium sulfide $\left(\mathrm{Li}_{2} \mathrm{~S}_{n}, 4<n<8\right)$, while $2.0 \mathrm{~V}$ convey to the arrangement of insoluble lithium sulfides $\left(\mathrm{Li}_{2} \mathrm{~S}_{2} / \mathrm{Li}_{2} \mathrm{~S}\right.$ ), respectively (Sun et al., 2008; Ji et al., 2011). There is only one main sharp oxidation peak observed in the anodic scan at around $2.47 \mathrm{~V}$, which indicates that multi lithium sulfide and $\mathrm{Li}_{2} \mathrm{~S}$ have oxidized into elemental sulfur. These outcomes endorse that there was no outstanding potential shift in the excellent electrochemical stability and also verifies the minimization of active loss in the as prepared sample
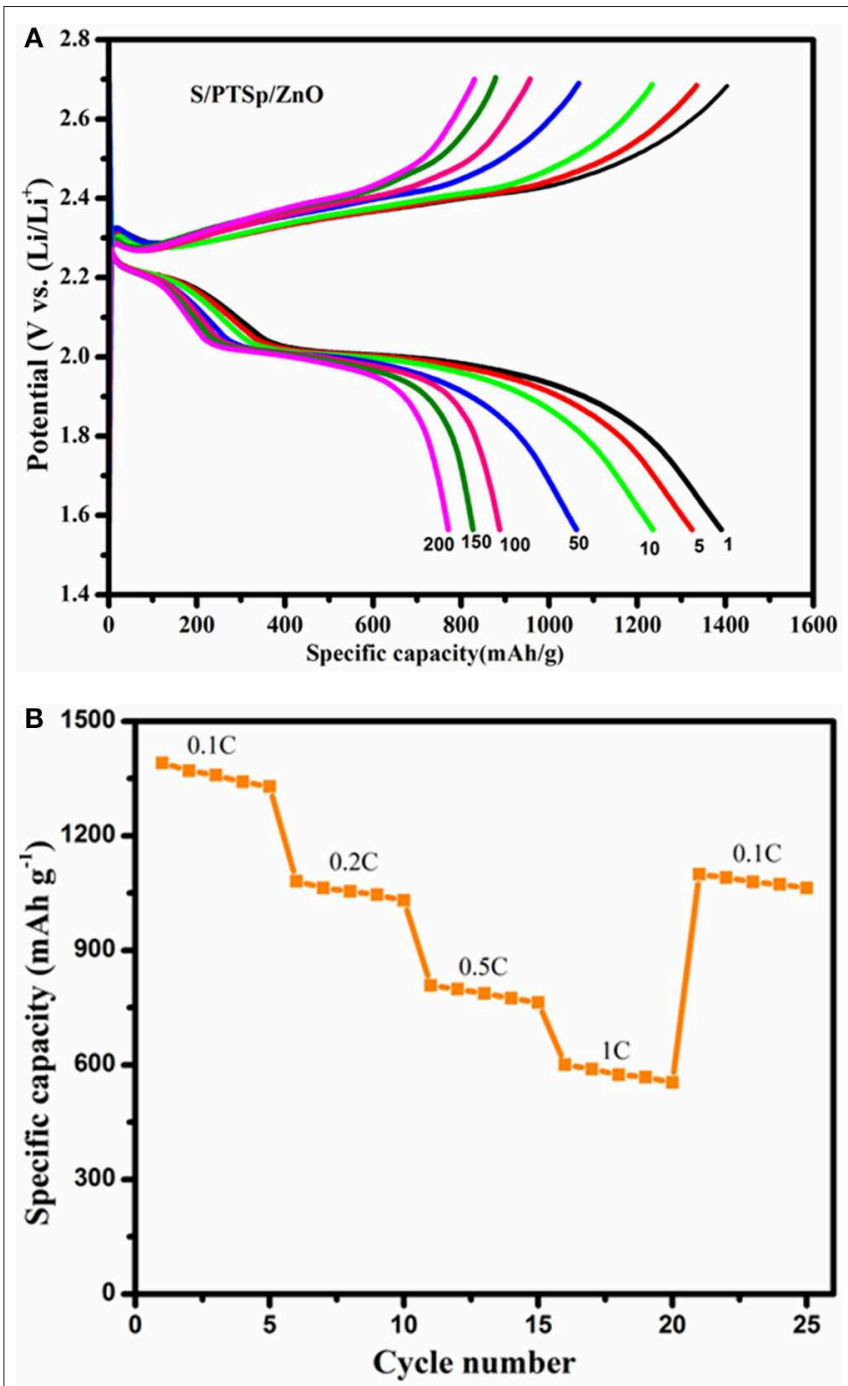

FIGURE 10 | (A) Galvanostatic charge-discharge curves of 1st, 5th, 10th, 50th, 100th, 150th, and 200th cycle of the S/PTSp/ZnO composite at a scan rate of 0.1 C. (B) Rate performance of the S/PTSp/ZnO.

and an improvement of reversibility of the anode with the cycling process.

Figure 10A shows the galvanostatic charge-discharge curves at different cycles for the S/PTSp/ZnO composite at a scan rate of $0.1 \mathrm{C}$ with the potential range of $1.5-2.7 \mathrm{~V}$. The specific capacity of the as prepared sample has been considered from the basis of sulfur mass (Lee et al., 2015). From the profile, it appears that there are two distinct discharge plateaus and one charge plateau was observed, which is consistent with the CV results (Zegeye et al., 2016). The initial specific discharge capacity of the S/PTSp/ZnO composite cathode material exhibits about 1,391 $\mathrm{mAhg}^{-1}$ and it maintains a $765 \mathrm{mAhg}^{-1}$ at 200th cycle. The sepiolite and $\mathrm{ZnO}$ nanoparticles are favorable for restraining the polysulfides in the cathode and avoiding their dissolution into electrolytes. The discharge potential plateaus of the S/PTSp/ZnO composite cathode are stable up to 50 cycles, which indicates 


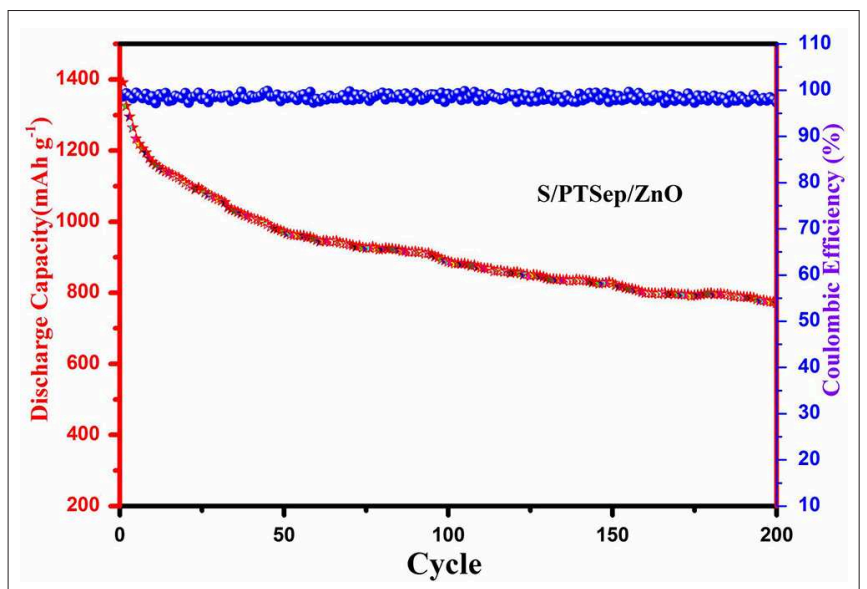

FIGURE 11 | Cyclic performance and the Coulombic efficiency of $\mathrm{S} / \mathrm{PTSp} / \mathrm{ZnO}$ Composites under discharge rate of $0.1 \mathrm{C}$.

the good electrochemical performance of the prepared composite cathode. Figure 10B demonstrates the rate performance of the $\mathrm{S} / \mathrm{PTSp} / \mathrm{ZnO}$ composite material at different current densities. By cycling from low to high rates of $0.1,0.2,0.5$, and $1 \mathrm{C}$, the composite electrode illustrated the charge capacities of 1,391 , $1,110,950$, and $751 \mathrm{mAhg}^{-1}$. The excellent cycling stability and rate capability are confirmed from the as-prepared sample.

Figure 11 shows the cyclic performance of the S/PTSp/ZnO composite material at a stable density of $0.1 \mathrm{C}$. The coulombic efficiency of $99 \%$ was obtained from the as-prepared sample, which showed an excellent reversible electrochemical reaction and also attained a capacity retention of about $69 \%$. The electrode material based on the high concentration of additive materials was used to recover the battery performance and also coulombic efficiency ensuring a drastically enhanced cycling stability, discharge capacity, and prevention of active material loss. As a result, the composite material exhibits a better performance due to a high content of additive sepiolite and metal oxide, which can strongly diffuse the polysulfide dissolution out of the electrode and improve the conductivity of the material.

\section{CONCLUSION}

To conclude, the Metal oxide $\mathrm{ZnO}$ nanoparticles were combined by co-precipitation technique and the sulfur/sepiolite/Metal oxide composite material was prepared using a simple heat

\section{REFERENCES}

Alvarez, A., Singer, A., and Galan, E. (1984). Sepiolite: properties and uses. Dev. Sedimentol. 37, 253-287. doi: 10.1016/S0070-4571(08)70044-X

Bokobza, L., Burr, A., Garnaud, G., Perrin, M. Y., and Pagnotta, S. (2004). Fibre reinforcement of elastomers: nanocomposites based on sepiolite and poly(hydroxyethyl acrylate). Polym. Int. 53, 1060-1065. doi: 10.1002/pi.1489

Bruce, P. G., Freunberger, S. A., Hardwick, L. J., and Tarascon, J. M. (2012). $\mathrm{Li}_{-} \mathrm{O}_{2}$ and Li-S batteries with high energy storage. Nat. Mater. 11, 19-29. doi: $10.1038 /$ nmat3191 treatment method. These examples were portrayed by XRD, FT-IR, RAMAN, TEM, XPS, TG, and SEM, examinations and electrochemical execution. All the diffraction peaks of the composites were in line with the standards. The functional vibrations of the as prepared composites were analyzed by FT-IR spectra. The fibrous morphology of the particles were observed by SEM images. In Raman spectra, below $500^{\circ} \mathrm{C}$ the sulfur and metal oxide peaks were obtained. TEM images clearly demonstrated the presence of sulfur, sepiolite, and $\mathrm{ZnO}$ nanoparticles. The above outcomes have clearly shown that the composite material is utilized to advance the great electrochemical execution of Lithium-sulfur batteries.

\section{DATA AVAILABILITY STATEMENT}

The raw data supporting the conclusions of this article will be made available by the authors, without undue reservation.

\section{AUTHOR CONTRIBUTIONS}

MS and RS: conceptualization, supervision, funding, methodology, investigation, formal analysis, original draft, review and editing, and project administration. CK: conceptualization, methodology, investigation, formal analysis, original draft, and review and editing. F-MW: conceptualization, funding, investigation, formal analysis, and review and editing.

\section{FUNDING}

All the authors acknowledge the financial support by DSTSERB, New Delhi under the Physical sciences, Grant sanctioned vide EMR/2016/006302. Also, all the authors acknowledge for the financial support by BSR of University grants Commission (UGC), FIST and PURSE programme of DST, New Delhi, India, and Ministry of Human Resource Development RUSA-Phase 2.0 Grant sanctioned vide Lt. No. F-24-51/2014U Policy (TN Multi Gen), Department of Education, Government of India.

\section{SUPPLEMENTARY MATERIAL}

The Supplementary Material for this article can be found online at: https://www.frontiersin.org/articles/10.3389/fmats. 2020.00109/full\#supplementary-material

Figure S1 | EDS analysis of the composite material.

Burzo, E., and Wijn, H. P. (2009). Phyllosilicates. Berlin; Heidelberg: SpringerVerlag, 340-403.

Can, M. F., Cinar, M., Benli, B., Ozdemir, O., and Celik, M. S. (2010). Determining the fiber size of nano structured sepiolite using Atomic Force Microscopy (AFM). Appl. Clay Sci. 47, 217-222. doi: 10.1016/j.clay.2009. 10.010

Coleman, V. A., and Jagadish, C. (2006). "Basic properties and applications of ZnO," in Zinc Oxide Bulk, Thin Films and Nanostructures, eds C. Jagadish and S. Pearton (Canberra, ACT: Australian National University; Elsevier), 1-20. doi: 10.1016/B978-008044722-3/50001-4 
Evers, S., and Nazar, L. F. (2013). New approaches for high energy density lithiumsulfur battery cathodes. Acc. Chem. Res. 46, 1135-1143. doi: 10.1021/ar3001348

$\mathrm{Fu}, \mathrm{M}$. Y., and Su, Y. S. (2013). Challenges and prospects of lithium-sulfur batteries. Acc. Chem. Res. 46, 1125-1134. doi: 10.1021/ar300179v

$\mathrm{Fu}$, R., Yang, Y., Xu, Z., Zhang, X., Guo, X., and Bi, D. (2015). The removal of chromium (VI) and lead (II) from groundwater using sepiolitesupported nanoscale zero-valent iron (S-NZVI). Chemosphere 138, 726-734. doi: 10.1016/j.chemosphere.2015.07.051

Gao, P. X., and Wang, Z. L. (2004). Nanopropeller arrays of zinc oxide. Appl. Phys Lett. 84, 2883-2885. doi: 10.1063/1.1702137

Han, S. C., Kim, K. W., Ahn, H. J., and Lee, J. Y. (2003). Chargedischarge mechanism of mechanically alloyed nis used as a cathode in rechargeable lithium batteries. J. Alloys Compd. 361, 247-251. doi: $10.1016 / 50925-8388(03) 00380-3$

Ji, L., Rao, M., Zheng, H., Zhang, L., Li, Y., Duan, W., et al. (2011). Graphene oxide as a sulfur immobilizer in high performance lithium/sulfur cells. J. Am. Chem. Soc. 133, 18522-18525. doi: 10.1021/ja206955k

Ji, X. L., and Nazar, L. F. (2010). Advances in Li-S batteries. J. Mater. Chem. 20, 9821-9826. doi: 10.1039/b925751a

Krishnaveni, K., Subadevi, R., Raja, M., Prem Kumar, T., and Sivakumar, M. (2018). Sulfur/PAN/acetylene black composite prepared by a solution processing technique for lithium-sulfur batteries. J. Appl. Polym. Sci. 135:46598. doi: $10.1002 /$ app. 46598

Kumar, P., Pournarab, A., Kim, K. H., Bansal, V., Rapti, S., and Manos, M. J. (2017). Challenges and opportunities for ion-exchange/sorption applications. Prog. Mater. Sci. 86, 25-74. doi: 10.1016/j.pmatsci.2017.01.002

Lee, J., Hwang, T., Lee, Y., Lee, J. K., and Choi, W. (2015). Coating of sulfur particles with manganese oxide nanowires as a cathode material in lithiumsulfur batteries. Mater. Lett. 158, 132-135. doi: 10.1016/j.matlet.2015.06.003

Li, F., Lu, W., Niu, S., and Li, B. (2014). Preparation and electrochemical performance of a graphene-wrapped carbon/sulphur composite cathode. New Carbon Mater. 29, 309-315. doi: 10.1016/S1872-5805(14)60140-2

Li, G. C., Hu, J. J., Li, G. R., Ye, S. H., and Gao, X. P. (2013). Sulfur/activatedconductive carbon black composites as cathode materials for lithium/sulfur battery. J. Power Sources 240, 598-605. doi: 10.1016/j.jpowsour.2013.02.095

Maensiri, S., Laokul, P., and Promarak, V. (2006). Synthesis and optical properties of nanocrystalline $\mathrm{ZnO}$ powders by a simple method using zinc acetate dihydrate and poly(vinyl pyrrolidone). J. Crystal Growth 289, 102-106. doi: $10.1016 /$ j.jcrysgro.2005.10.145

Moezzi, A., McDonagh, A. M., and Cortie, M. B. (2012). Zinc oxide particles: synthesis, properties and applications. Chem. Eng. J. 185-186, 1-22. doi: 10.1016/j.cej.2012.01.076

Muruganantham, R., Sivakumar, M., and Subadevi, R. (2016). Synthesis and electrochemical characterization of olivine-type lithium iron phosphate cathode materials via different techniques. Ionics. 22, 1557-1565. doi: 10.1007/s11581-016-1676-3

Ozgur, U., Alivov Ya,. I., Liu, C., Teke, A., Reshchikov, M. A., Dogan, S., et al. (2005). A comprehensive review of $\mathrm{ZnO}$ materials and devices. J. Appl. Phys. 98, 041301-1-041301-101. doi: 10.1063/1.1992666

Qiu, L. L., Zhang, S. C., Zhang, L., Sun, M. M., and Wang, W. K. (2010). Preparation and enhanced electrochemical properties of nanosulfur/poly(pyrrole-co-aniline) cathode material for lithium/sulfur batteries. Electrochim. Acta 55, 4632-4636. doi: 10.1016/j.electacta.2010.03.030

Radhika, G., Krishnaveni, R., Liu, W. R., and Sivakumar, M. (2018). Synthesis and electrochemical performance of PEG- $\mathrm{MnO}_{2}$-sulfur composites cathode materials for lithium-sulfur batteries. J. Nanosci. Nanotechnol. 18, 127-131. doi: $10.1166 /$ jnn.2018.14568
Rajkumar, P., Diwakar, K., Radhika, G., Krishnaveni, K., Subadevi, R., and Sivakumar, M. (2018). Effect of silicon dioxide in sulfur/carbon black composite as a cathode material for lithium sulfur batteries. Vacuum 161, 37-48. doi: 10.1016/j.vacuum.2018.12.016

Song, M. S., Han, S. C., Kim, H. S., Kim, J. H., Kim, K. T., Kang, Y. M., et al. (2004). Effects of nanosized adsorbing material on electrochemical properties of sulfur cathodes for Li/S secondary batteries. J. Electrochem. Soc. A 151:791. doi: $10.1149 / 1.1710895$

Sun, M., Zhang, S., Jiang, T., Zhang, L., and Yu, J. (2008). Nanowire networks of sulfur-polypyrrole composite cathode materials for rechargeable lithium batteries. Electrochem. Commun. 10, 1819-1822. doi: 10.1016/j.elecom.2008.09.012

Sun, X. C., Zhang, H. Z., Xu, J., Zhao, Q., Wang, R. M., and Yu, D. P. (2004). Shape controllable synthesis of $\mathrm{ZnO}$ nanorod arrays via vapor phase growth. Solid State Commun. 129, 803-807. doi: 10.1016/j.ssc.2003.11.051

Vayssieres, L. (2003). Growth of arrayed nanorods and nanowires of $\mathrm{ZnO}$ from aqueous solutions. Adv. Mater. 15, 464-466. doi: 10.1002/adma.2003 90108

Ward, A. T. (1968). Raman spectroscopy of sulfur, sulfur-selenium, and sulfurarsenic mixtures. J. Phys. Chem. 72, 4133-4139. doi: 10.1021/j100858a031

Wu, F., Chen, J., Chen, R., Wu, S., Li, L., Chen, S., et al. (2011). Sulfur/polythiophene with a core/shell structure: synthesis and electrochemical properties of the cathode for rechargeable lithium batteries. J. Phys. Chem. C 115, 6057-6063. doi: 10.1021/jp1114724

Xu, Y., Wen, Y., Zhu, Y., Gaskell, K., Cychosz, K. A., Eichhorn, B., et al. (2015). Confined sulfur in microporous carbon renders superior cycling stability in Li/S batteries. Adv. Funct. Mater. 25, 4312-4320. doi: 10.1002/adfm.2015 00983

Yang, X., Zhu, W., Cao, G., and Zhao, X. (2015). Preparation of reduced carbon-wrapped carbon-sulfur composite as cathode material of lithiumsulfur batteries. RSC Adv. 5, 93926-93936. doi: 10.1039/C5RA20262K

Zegeye, T. A., Kuo, C. J., Wotango, A. S., Pan, C., Chen, H., Haregewoin, A. M., et al. (2016). Hybrid nanostructured microporous carbon-mesoporous carbon doped titanium dioxide/sulfur composite positive electrode materials for rechargeable lithium-sulfur batteries. J. Power Sources 324, 239-252. doi: 10.1016/j.jpowsour.2016.05.080

Zhang, H., Ma, X. Y., Xu, J., Niu, J., and Yang, D. (2003). Arrays of ZnO nanowires fabricated by a simple chemical solution route. Nanotechnology 14, 423-426. doi: 10.1088/0957-4484/14/4/303

Zhang, J., Zhu, Y., Cao, C., and Butt, F. K. (2015). Microwave-assisted and largescale synthesis of $\mathrm{SnO}_{2}$ /carbon-nanotube hybrids with high lithium storage capacity. RSC Adv. 5, 58568-58573. doi: 10.1039/C5RA10314B

Zheng, W., Hu, X. G., and Zhang, C. F. (2006). Electrochemical properties of rechargeable lithium batteries with sulfur-containing composite cathode materials. Electrochem. Solid State Lett. 9:364. doi: 10.1149/1.2203348

Conflict of Interest: The authors declare that the research was conducted in the absence of any commercial or financial relationships that could be construed as a potential conflict of interest.

Copyright () 2020 Kalaiselvi, Subadevi, Wang and Sivakumar. This is an open-access article distributed under the terms of the Creative Commons Attribution License (CC $B Y)$. The use, distribution or reproduction in other forums is permitted, provided the original author(s) and the copyright owner(s) are credited and that the original publication in this journal is cited, in accordance with accepted academic practice. No use, distribution or reproduction is permitted which does not comply with these terms. 